\title{
ALGEBRAIC VALUES OF TRANSCENDENTAL FUNCTIONS AT ALGEBRAIC POINTS
}

\author{
JINGJING HUANG, DIEGO MARQUES ${ }^{\bowtie}$ and MARTIN MEREB
}

(Received 20 January 2010)

\begin{abstract}
It is shown that any subset of $\overline{\mathbb{Q}}$ can be the exceptional set of some transcendental entire function. Furthermore, we give a much more general version of this theorem and present a unified proof.
\end{abstract}

2000 Mathematics subject classification: primary 11J81.

Keywords and phrases: exceptional set, transcendental, algebraic.

\section{Introduction}

In 1886, Weierstrass gave an example of a transcendental entire function which takes rational values at all rational points. He also suggested that there exist transcendental entire functions which take algebraic values at any algebraic point. Later, in [3], Stäckel proved that for each countable subset $\Sigma \subseteq \mathbb{C}$ and each dense subset $T \subseteq \mathbb{C}$, there is a transcendental entire function $f$ such that $f(\Sigma) \subseteq T$. Another construction due to Stäckel produces an entire function $f$ whose derivatives $f^{(s)}$, for $s=0,1,2, \ldots$, all map $\overline{\mathbb{Q}}$ into $\overline{\mathbb{Q}}$; see [4]. A more thorough discussion on this subject can be found in $[2,6]$. There are recent results due to Surroca on the number of algebraic points where a transcendental analytic function takes algebraic values, see [5]. We were able to generalize these two results of Stäckel to the following general theorem.

THEOREM 1. Given a countable subset $A \subseteq \mathbb{C}$ and for each integer $s \geq 0$ with $\alpha \in A$, fix a dense subset $E_{\alpha, s} \subseteq \mathbb{C}$. Then there exists a transcendental entire function $f: \mathbb{C} \rightarrow \mathbb{C}$ such that $f^{(s)}(\alpha) \in E_{\alpha, s}$, for all $\alpha \in A$ and all $s \geq 0$.

Let $f$ be given, and denote by $S_{f}$ the set of all algebraic points $\alpha \in \mathbb{C}$, for which $f(\alpha)$ is also algebraic. An interesting problem is to determine properties of $S_{f}$, which we call the exceptional set of $f$. In the conclusion we will show that for any $A \subseteq \overline{\mathbb{Q}}$ there is a transcendental entire function $f$ such that $A$ is the exceptional set of $f$.

Without referring to Theorem 1, we have the following special examples.

The second author is partially supported by FEMAT. The third author is supported by a Harrington Fellowship.

(C) 2010 Australian Mathematical Publishing Association Inc. 0004-9727/2010 \$16.00 
EXAMPLE 2. Arbitrary finite subsets of algebraic numbers are easily seen to be exceptional. For instance, if $f_{1}(z)=e^{\left(z-\alpha_{1}\right) \cdots\left(z-\alpha_{k}\right)}$, then the Hermite-Lindemann theorem implies that $S_{f_{1}}=\left\{\alpha_{1}, \ldots, \alpha_{k}\right\}$. If $f_{2}(z)=e^{z}+e^{z+1}$ and $f_{3}(z)=e^{z \pi+1}$, then the Lindemann-Weierstrass and Baker theorems imply that $S_{f_{2}}=S_{f_{3}}=\emptyset$.

EXAMPlE 3. Some well-known infinite sets are also exceptional; for instance, if $f_{4}(z)=2^{z}, f_{5}(z)=e^{i \pi z}$, then $S_{f_{4}}=S_{f_{5}}=\mathbb{Q}$, by the Gelfond-Schneider theorem.

EXAMPLE 4. Assuming Schanuel's conjecture to be true, it is easy to prove that if

$$
f_{6}(z)=\sin (\pi z) e^{z}, \quad f_{7}(z)=2^{3^{z}} \text { and } \quad f_{8}(z)=2^{2^{2^{z-1}}}
$$

then $S_{f_{6}}=S_{f_{7}}=\mathbb{Z}$ and $S_{f_{8}}=\mathbb{N}$.

These examples are just special cases of our Theorem 1; they can be proved uniformly here.

\section{Preliminary results}

In order to prove Theorem 1, we need several lemmas.

LEMMA 5. Let $\left\{P_{n}(z)\right\}_{n \geq 0}$ be a sequence of complex polynomials, where $\operatorname{deg} P_{n}=$ n. Also let $\left\{C_{n}\right\}_{n \geq 0}$ be a sequence of positive constants such that $\left|P_{n}(z)\right| \leq$ $C_{n} \max \{|z|, 1\}^{n}$. If a sequence of complex numbers $\left\{a_{n}\right\}_{n \geq 0}$ satisfies $\left|a_{n}\right| \leq 1 / C_{n} n$ !, then the series $\sum_{n=0}^{\infty} a_{n} P_{n}(z)$ converges absolutely and uniformly on any compact set; in particular, this gives an entire function.

Proof. When $\left|a_{n}\right| \leq 1 / C_{n} n$ !,

$$
\sum_{n=0}^{\infty}\left|a_{n}\right|\left|P_{n}(z)\right| \leq \sum_{n=0}^{\infty} \frac{1}{C_{n} n !} C_{n} \max \{|z|, 1\}^{n} \leq \exp (\max \{|z|, 1\}),
$$

so $\sum_{n=0}^{\infty} a_{n} P_{n}(z)$ converges absolutely and uniformly on any compact set. Therefore this series will produce an entire function.

Let us now enumerate the set $A$ in Theorem 1 as $\left\{\alpha_{1}, \alpha_{2}, \alpha_{3}, \ldots\right\}$.

For $n \geq 1$, define $m_{n}$ and $j_{n}$ by $n=1+2+3+\cdots+m_{n}+j_{n}$, where $m_{n} \geq 0$ and $1 \leq j_{n} \leq m_{n}+1$. Next, construct a sequence of polynomials by letting $P_{0}(z)=1$ and defining recursively

$$
P_{n}(z)=\left(z-\alpha_{j_{n}}\right) P_{n-1}(z) \text { for } n \geq 1 .
$$

Here we list the first few polynomials:

$$
\begin{aligned}
& P_{0}(z)=1 \\
& P_{1}(z)=\left(z-\alpha_{1}\right) \\
& P_{2}(z)=\left(z-\alpha_{1}\right)^{2} \\
& P_{3}(z)=\left(z-\alpha_{1}\right)^{2}\left(z-\alpha_{2}\right) \\
& P_{4}(z)=\left(z-\alpha_{1}\right)^{3}\left(z-\alpha_{2}\right)
\end{aligned}
$$




$$
\begin{aligned}
& P_{5}(z)=\left(z-\alpha_{1}\right)^{3}\left(z-\alpha_{2}\right)^{2} \\
& P_{6}(z)=\left(z-\alpha_{1}\right)^{3}\left(z-\alpha_{2}\right)^{2}\left(z-\alpha_{3}\right) \\
& P_{7}(z)=\left(z-\alpha_{1}\right)^{4}\left(z-\alpha_{2}\right)^{2}\left(z-\alpha_{3}\right)
\end{aligned}
$$

Let $i_{n}=m_{n}+1-j_{n}$. For any given $i \geq 0$ and $j \geq 1$ there exists a unique $n \geq 1$ such that $i_{n}=i$ and $j_{n}=j$, namely $n=\frac{1}{2}(i+j)(i+j-1)+j$.

LEMMA 6. For $n \geq 1$, we have $P_{n-1}^{\left(i_{n}\right)}\left(\alpha_{j_{n}}\right) \neq 0$ and $P_{l}^{\left(i_{n}\right)}\left(\alpha_{j_{n}}\right)=0$ when $l \geq n$.

PROOF. From the definition of $P_{n}(z)$, we can write explicitly

$$
P_{l}(z)=\left(z-\alpha_{1}\right)^{m_{l}}\left(z-\alpha_{2}\right)^{m_{l}-1} \cdots\left(z-\alpha_{m_{l}}\right)\left(z-\alpha_{1}\right) \cdots\left(z-\alpha_{j_{l}}\right) .
$$

It follows that $\alpha_{j_{n}}$ is a zero of $P_{n-1}(z)$ with multiplicity $i_{n}$, which means that $P_{n-1}^{\left(i_{n}\right)}\left(\alpha_{j_{n}}\right) \neq 0$. On the other hand, if $l \geq n$, then $\alpha_{j_{n}}$ is a zero of $P_{l}(z)$ with multiplicity at least $i_{n}+1$, which implies that $P_{l}^{\left(i_{n}\right)}\left(\alpha_{j_{n}}\right)=0$.

LEMMA 7. If $\sum_{k=0}^{\infty} a_{k} P_{k}(z)=\sum_{k=0}^{\infty} b_{k} P_{k}(z)$ for all $z \in \mathbb{C}$, then $a_{k}=b_{k}$ for each $k \geq 0$.

PROOF. It suffices to prove that if $g(z):=\sum_{k=0}^{\infty} a_{k} P_{k}(z)=0$ for all $z \in \mathbb{C}$, then $\left\{a_{k}\right\}_{k \geq 0}$ is identically 0 . Notice that $a_{0}=g\left(\alpha_{1}\right)=0$. Assuming that $a_{0}, a_{1}, \ldots, a_{n-1}$ are all 0 , by Lemma 6 ,

$$
\begin{aligned}
0 & =\sum_{k=0}^{\infty} a_{k} P_{k}^{\left(i_{n+1}\right)}\left(\alpha_{j_{n+1}}\right) \\
& =\sum_{k=0}^{n-1} a_{k} P_{k}^{\left(i_{n+1}\right)}\left(\alpha_{j_{n+1}}\right)+a_{n} P_{n}^{\left(i_{n+1}\right)}\left(\alpha_{j_{n+1}}\right)+\sum_{k=n+1}^{\infty} a_{k} P_{k}^{\left(i_{n+1}\right)}\left(\alpha_{j_{n+1}}\right) \\
& =a_{n} P_{n}^{\left(i_{n+1}\right)}\left(\alpha_{j_{n+1}}\right) .
\end{aligned}
$$

Since $P_{n}^{\left(i_{n+1}\right)}\left(\alpha_{j_{n+1}}\right) \neq 0$, we have $a_{n}=0$. The proof is completed by induction.

We are now able to prove our theorem.

\section{Proof of Theorem 1}

We will construct the desired transcendental entire function by fixing the coefficients in the series $\sum_{k=0}^{\infty} a_{k} P_{k}(z)$ recursively, where the sequence $\left\{P_{k}\right\}_{k \geq 0}$ has been defined in Section 2.

First, by Lemma 5 , the condition $\left|a_{k}\right| \leq 1 / C_{k} k$ ! will ensure that $\sum_{k=0}^{\infty} a_{k} P_{k}(z)$ is entire. 
Now we will fix the coefficients $a_{k}$ recursively. For $n \geq 1$, we denote $E_{n}=E_{\alpha_{j_{n}}, i_{n}}$ and let the numbers $\beta_{n}=\sum_{k=0}^{\infty} a_{k} P_{k}^{\left(i_{n}\right)}\left(\alpha_{j_{n}}\right)$. We will choose the value of $a_{k}$ so that $\beta_{n} \in E_{\alpha_{j}, i_{n}}=E_{n}$ for all $n \geq 1$.

By Lemma 6 , we know that $P_{l}^{\left(i_{n}\right)}\left(\alpha_{j_{n}}\right)=0$ when $l \geq n$, so $\beta_{n}$ is actually the finite $\operatorname{sum} \sum_{k=0}^{n-1} a_{k} P_{k}^{\left(i_{n}\right)}\left(\alpha_{j_{n}}\right)$. Notice that $\beta_{1}=a_{0} P_{0}^{(0)}\left(\alpha_{1}\right)=a_{0}$ and $E_{1}$ is dense; we can fix a value for $a_{0}$ such that $0<\left|a_{0}\right| \leq 1 / C_{0}$ and $\beta_{1} \in E_{1}$. Now suppose that the values of $\left\{a_{0}, a_{1}, \ldots, a_{n-1}\right\}$ are well fixed such that $0<\left|a_{k}\right| \leq 1 / C_{k} k$ ! and $\beta_{k} \in E_{k}$ for $0 \leq k \leq n-1$. By Lemma 6 , we know $P_{n}^{\left(i_{n+1}\right)}\left(\alpha_{j_{n+1}}\right) \neq 0$, so we can pick a proper value of $a_{n}$ such that $0<\left|a_{n}\right| \leq 1 / C_{n} n$ ! and

$$
\beta_{n}=\sum_{k=0}^{n-1} a_{k} P_{k}^{\left(i_{n+1}\right)}\left(\alpha_{j_{n+1}}\right)+a_{n} P_{n}^{\left(i_{n+1}\right)}\left(\alpha_{j_{n+1}}\right) \in E_{n} .
$$

So now by induction all the $a_{k}$ are well chosen such that for all $k \geq 1$ we have $0<$ $\left|a_{k}\right| \leq 1 / C_{k} k$ ! and $\beta_{k} \in E_{k}$. Thus by Lemma 5, the function $f(z)=\sum_{k=0}^{\infty} a_{k} P_{k}(z)$ is entire and for any $i \geq 0, j \geq 1$ we have $f^{(i)}\left(\alpha_{j}\right)=\sum_{k=0}^{\infty} a_{k} P_{k}^{(i)}\left(\alpha_{j}\right)=\beta_{n} \in E_{n}=$ $E_{\alpha_{j}, i}$ where $n$ is the unique integer such that $i_{n}=i, j_{n}=j$. Taking into account that every polynomial can be expressed as a finite linear combination of the $\left\{P_{k}\right\}$, and all the $\left\{a_{k}\right\}$ here are not 0 , so by Lemma 7 we conclude that $f(z)$ is not a polynomial. Hence $f(z)$ is the desired transcendental entire function, and the proof is complete.

From the construction of the proof, we can easily see that in fact there are uncountably many functions satisfying the properties required in Theorem 1 .

\section{Applications to exceptional sets}

We recall the following definition.

DEFINITION 8. Let $f$ be an entire function. We define the exceptional set of $f$ to be

$$
S_{f}=\{\alpha \in \overline{\mathbb{Q}} \mid f(\alpha) \in \overline{\mathbb{Q}}\} .
$$

We list some of the more interesting consequences of Theorem 1 with the choice of $A, E_{\alpha, s}$ noted in parentheses.

COROllary 9. For each countable subset $\Sigma \subseteq \mathbb{C}$ and for each dense subset $T \subseteq \mathbb{C}$ there is a transcendental entire function $f$ such that $f^{(s)}(\Sigma) \subseteq T$ for $s \geq 0$. $(A=\Sigma$, $E_{\alpha, s}=T$.)

Corollary 10. Let $A \subseteq \mathbb{C}$ be countable and dense in $\mathbb{C}$. Then there is a transcendental entire function $f$ such that $f^{(s)}(A) \subseteq A$, for $s \geq 0$. $\left(E_{\alpha, s}=A\right.$.)

COROLlaRY 11. There exists a transcendental entire function such that $f^{(s)}(\overline{\mathbb{Q}}) \subseteq$ $\mathbb{Q}(i)$, for $s \geq 0 .\left(A=\overline{\mathbb{Q}}, E_{\alpha, s}=\mathbb{Q}(i)\right.$.) 
A set $A$ is said to be closed (with respect to $\overline{\mathbb{Q}}$ ) if it has the following property: if $\alpha$ is algebraic and $\alpha^{\prime}$ is any algebraic conjugate of $\alpha$, then $\alpha \in A$ implies that also $\alpha^{\prime} \in A$. In 1965, Mahler [1] proved that every closed set is the exceptional set of some transcendental entire function. Our next result shows, in particular, that another interesting consequence of Theorem 1 is that every $A \subseteq \overline{\mathbb{Q}}$ is an exceptional set of a transcendental entire function.

THEOREM 12. If $A \subseteq \overline{\mathbb{Q}}$, then there is a transcendental entire function such that $S_{f^{(s)}}=A$ for $s \geq 0$.

Proof. Suppose that $A$ and $\overline{\mathbb{Q}} \backslash A$ are both infinite, thus we can enumerate $\overline{\mathbb{Q}}=\left\{\alpha_{1}, \alpha_{2}, \ldots\right\}$ where $A=\left\{\alpha_{1}, \alpha_{3}, \ldots, \alpha_{2 n+1}, \ldots\right\}$. Set $E_{\alpha_{2 n+2}, s}=\mathbb{C} \backslash \overline{\mathbb{Q}}$ and $E_{\alpha_{2 n+1}, s}=\overline{\mathbb{Q}}$ for all $n, s \geq 0$. Now by Theorem 1 , there exists a transcendental entire function $f$ with $f^{(s)}\left(\alpha_{2 n+1}\right) \in \overline{\mathbb{Q}}$ and $f^{(s)}\left(\alpha_{2 n+2}\right) \in \mathbb{C} \backslash \overline{\mathbb{Q}}$, for each $n, s \geq 0$. Therefore it is plain that $S_{f(s)}=A$.

For the case where $A$ is finite, we can suppose that $A=\left\{\alpha_{1}, \ldots, \alpha_{m}\right\}$. Take $E_{\alpha_{1}, s}=\cdots=E_{\alpha_{m}, s}=\overline{\mathbb{Q}}$ for all $s \geq 0$, and set $E_{\alpha_{k}, s}=\mathbb{C} \backslash \overline{\mathbb{Q}}$ for all $k>m, s \geq 0$. If $\overline{\mathbb{Q}} \backslash A=\left\{\alpha_{1}, \ldots, \alpha_{m}\right\}$, we take $E_{\alpha_{1}, s}=\cdots E_{\alpha_{m}, s}=\mathbb{C} \backslash \overline{\mathbb{Q}}$ for all $s \geq 0$, and set $E_{\alpha_{k}, s}=\overline{\mathbb{Q}}$ for all $k>m, s \geq 0$. Then for these two cases we proceed as in the proof above.

\section{Acknowledgements}

The authors would like to express their gratitude to Professor Michel Waldschmidt for his instruction and guidance. We also thank Georges Racinet for providing encouragement and assistance during the evening workshops that took place at the 2008 Arizona Winter School. Additionally, we owe a debt to all of the students who participated in those workshops: Chuangxun Cheng, Brian Dietel, Mathilde Herblot, Holly Krieger, Jonathan Mason and Robert Wilson. Finally, we must thank the organizers of the Arizona Winter School and the hospitality of the University of Arizona for making this work possible.

\section{References}

[1] K. Mahler, 'Arithmetic properties of lacunary power series with integral coefficients', J. Aust. Math. Soc. 168 (1965), 200-227.

[2] K. Mahler, Lectures on Transcendental Numbers, Lecture Notes in Mathematics, 546 (Springer, Berlin, 1976).

[3] P. Stäckel, 'Ueber arithmetische Eigenschaften analytischer Functionen', Math. Ann. 46(4) (1895), 513-520.

[4] P. Stäckel, 'Arithmetische Eigenschaften analytischer Functionen', Acta Math. 25 (1902), 371-383.

[5] A. Surroca, 'Valeurs algébriques de fonctions transcendantes', Int. Math. Res. Not. (2006), Art. ID $16834,31 \mathrm{pp}$.

[6] M. Waldschmidt, 'Algebraic values of analytic functions', Proceedings of the International Conference on Special Functions and their Applications (Chennai, 2002), J. Comput. Appl. Math. 160(1-2) (2003), 323-333. 
JINGJING HUANG, Mathematics Department, Pennsylvania State University, PA 16802, USA

e-mail: huang@math.psu.edu

DIEGO MARQUES, Departamento De Matemática, Universidade de Brasília, Brasília, CEP: 70910-900 DF, Brazil

e-mail: diego@mat.unb.br

MARTIN MEREB, Mathematics Department, University of Texas at Austin, TX 78712, USA

e-mail: mmereb@gmail.com 